\title{
Temporal Spectrum Sharing based on Primary User Activity Prediction
}

\author{
Ki Won Sung, Seong-Lyun Kim, and Jens Zander
}

\begin{abstract}
In this paper we investigate the opportunistic spectrum access in temporal domain where a secondary user shares a radio channel with a primary user during the OFF period of the primary user. We consider practical ON/OFF traffic models whose bursty natures are not properly described by a Markovian assumption. An optimal strategy to determine the transmission power of the secondary user is proposed, which can be adapted to any source traffic model of the primary user. This strategy will maximize the spectrum utilization of the secondary user while keeping interference violations to the primary user below a threshold. Numerical results show that the transmission power of the secondary user depends on the probability distribution of the primary traffic as well as the elapsed time of the OFF period.
\end{abstract}

Index Terms-Opportunistic spectrum access, temporal spectrum sharing, transmission power control, traffic model

\section{INTRODUCTION}

$\mathbf{R}$ ADIO spectrum has become a scarce resource with the rapid growth of the demand for wireless communications. The need for the spectrum resource is expected to increase more as pervasive wireless services and applications are likely to prevail in the near future. In contrast to the apparent shortage of spectrum, measurement results for the spectrum usage suggest that only a fraction of the spectrum is actually used at any given time and location [1], [2]. This discrepancy between the spectrum demand and the actual utilization necessitates more efficient methods of exploiting the spectrum. One promising solution is the opportunistic spectrum access (OSA) that is envisioned by the broader concept of cognitive radio [3], [4]. The OSA allows secondary (cognitive) users to share radio channels with primary (legacy) users provided that the interference caused by the secondary users is not intrusive to the primary users.

The spectrum opportunity can be discovered in spatial and temporal domains. The spatial aspect of OSA relies on the attenuation of radio signal which enables an enough separation between a secondary user and a primary user to prevent harmful interference. The capacity of the spatial OSA has been extensively studied. The capacity limit of the spectrum sharing

This work was supported by the VINNOVA project Multi-Operator Dynamic Spectrum Access (MODyS). The second author acknowledges that this research was supported by the International Research \& Development Program of the National Research Foundation of Korea (NRF) funded by the Ministry of Education, Science and Technology (MEST) of Korea (K2090100140609B1300-03110, FY 2010).

K. W. Sung and J. Zander are with Wireless@KTH, Royal Institute of Technology (KTH), SE 164 40, Kista, Sweden (e-mail: sungkw@kth.se, jenz@kth.se).

S-.L. Kim is with School of Electrical and Electronic Engineering, Yonsei University, Seoul 120-749, Korea (e-mail: slkim@yonsei.ac.kr). was investigated in [5]. The impact of aggregate interference induced by multiple secondary users was examined in [6], [7]. In [8], spectrum sharing in a frequency-planned cellular environment was evaluated. Reliable detection of the primary user is crucial in recognizing and exploiting the spatial opportunity. Performance of various sensing schemes to detect spatial opportunities were compared in a realistic propagation environment in [9]. An extensive survey of OSA can be found in [10].

This paper focuses on temporal aspect of the spectrum sharing. The temporal OSA exploits the bursty nature of the data traffic. A primary user alternates ON (busy) and OFF (idle) states during its service time. The channel becomes available to a secondary user when the primary traffic is idle even if the primary user is in close proximity to the secondary user. In [11]-[13], channel selection/switching problems were investigated under multiple ON/OFF channel environments aiming at minimizing the disruption to primary users. The authors of [14] examined the impact of primary ON/OFF traffic on the capacity of spectrum sharing. Scheduling of spectrum sensing interval was studied in [15]-[17], where the tradeoff between sensing efficiency and interference to primary users was addressed by dynamically adjusting the inter-sensing duration. However, the flexible sensing interval may introduce a synchronization problem to the secondary transmitter and receiver pair.

In this paper, we consider a fixed-length frame structure for the secondary user to help a simple synchronization. The frame is assumed to consist of multiple time slots. We propose an optimal power control scheme for the secondary user. At the beginning of each frame, the secondary user decides whether to transmit or not for the remaining time slots in the frame. The decision is based on the prediction of the primary user activity, i.e. the expected remaining time of the OFF period. The objective of the proposed scheme is twofold: to maximize the spectrum utilization of the secondary user and to keep the interference perceived by the primary user below a threshold.

It is known that the burstiness of the wireless source traffic is not properly described by the exponential distribution [18], [19]. Thus, we consider practical non-Markovian source traffic models. Peer-to-peer (P2P) and interactive gaming services are assumed for the primary user traffic in this study because these are promising applications for the wireless Internet [20][22]. Interestingly, these applications show opposite statistical behaviors in terms of the remaining time of the OFF period. The framework discussed in this paper can be readily adapted to any source traffic model. Numerical experiments are performed with the consideration of the detection errors and 
the inaccuracy in the traffic parameter estimation. Our results give new insights on how the non-Markovian behavior of the primary user makes an impact on the temporal opportunity of the spectrum.

The paper is organized as follows: In Section II, the system model is explained and the problem is described. The optimal transmission strategy for the secondary user is derived in Section III. Then, numerical results are presented in Section IV. Finally, conclusions are drawn in Section V.

\section{System Model And Problem Description}

\section{A. System Model}

A pair of primary users and a pair of secondary users are assumed to be located in a same transmission area. A wireless channel is shared by the primary and the secondary users. We assume the secondary user always has data to transmit. The channel access of the secondary user can be neither regular nor planned in advance since it depends on the activity of the primary user. Thus, synchronization between the secondary transmitter and receiver is an important implementation issue in temporal spectrum sharing. We assume a fixed-length frame structure for the secondary user to enable a simple synchronization. The frame consists of $N+1$ time slots, where the first slot is dedicated to periodic spectrum sensing. The remaining $N$ slots are for either transmission or shut down depending on the prediction of the primary user activity. Out of $N$ slots, those of no transmission can also be used for the spectrum sensing for more accurate knowledge of the primary user activity. This additional sensing may be used to find out when the OFF duration of the primary user started.

The source traffic of the primary user alternates ON (busy) and OFF (idle) periods. Let $D_{\text {off }}$ be a random variable denoting the duration of the OFF period. The probability distribution of $D_{\text {off }}$ depends on the specific application that the primary user currently employs. The cumulative distribution function $(\mathrm{CDF})$ of $D_{\text {off }}$ is denoted by $F_{\text {off }}(\cdot)$. A transmission by the secondary user causes an interference violation if the primary user is $\mathrm{ON}$ at the same time. The threshold of the interference violation probability is denoted by $p_{t h r}$.

\section{B. Formal Problem Description}

The moment that the primary user begins an OFF period is regarded as the time slot one. Then, $\tau$ denotes the elapsed time of the OFF period. Let us consider a frame starting from the time slot $\tau$ and suppose the secondary user perceives the primary user is still idle. At time slot $\tau$, the secondary user determines its transmission power for the next $N$ time slots in the frame, i.e. it decides whether to transmit or not for each time slot. A conservative decision under-utilizes the temporal opportunity, whereas an aggressive transmission strategy leads to the interference violation as illustrated in Fig. 1.

The objective of the secondary user is to maximize spectrum utilization while keeping the interference violation probability below $p_{t h r}$ during the frame duration. Let $I_{s u}(t)$ be a binary variable indicating whether the secondary user transmits at time slot $t$. By defining $I_{i v}(t)$ as an indicator variable for the interference violation at $t$, our problem can be written as follows:

$$
\max \sum_{t=\tau+1}^{\tau+N} I_{s u}(t),
$$

subject to

$$
\sum_{t=\tau+1}^{\tau+N} I_{i v}(t) \leq N p_{t h r} .
$$

Note that $I_{i v}(t)$ can be expressed as

$$
I_{i v}(t)=I_{p u}(t) I_{s u}(t),
$$

where $I_{p u}(t)$ is a variable indicating the activity of the primary user.

The secondary user can exploit the channel if the primary user is OFF $\left(I_{p u}(t)=0\right)$. It should stop transmitting when the primary user becomes $\mathrm{ON}\left(I_{p u}(t)=1\right)$. However, the secondary user is unable to know $I_{p u}(t)$ at the moment of decision $\tau(t>\tau)$. Thus, the transmission power of the secondary user should be determined based on the prediction of $I_{p u}(t)$ that depends on the specific probability distribution of the primary source traffic and the elapsed time of the OFF period. This is discussed in the next section.

\section{Optimal Transmission Strategy BASED ON PRIMARY TRAFFIC MODEL}

\section{A. Optimal Strategy}

Let us assume $n_{\tau}$ slots are transmitted out of $N$ time slots and $N-n_{\tau}$ slots are not used for the transmission in the considered frame, i.e. $\sum_{t=\tau+1}^{\tau+N} I_{s u}(t)=n_{\tau}$. Then, we propose the following transmission strategy for the secondary user: it transmits data in $n_{\tau}$ consecutive slots followed by $N-n_{\tau}$ slots of no transmission.

The proposed strategy has an advantage in synchronization and receiver complexity compared to other possible strategies spreading $n_{\tau}$ slots discontinuously over the frame. It allows the secondary user to commence transmission only at the second time slot of each frame. In addition to the simplicity, the strategy has the following proposition.

Proposition 1: The proposed strategy is optimal under the condition that the ON period of the primary user is longer than the frame length of the secondary user.

Proof: Assume that there is an optimal strategy which does not transmit in $j^{\text {th }}$ time slot and transmits in $k^{\text {th }}$ slot $(j<k)$. If the OFF period ends between $j+1^{\text {th }}$ and $k^{\text {th }}$ time slots, this strategy results in more interference violations than a strategy that exchanges $j^{\text {th }}$ and $k^{\text {th }}$ time slots. This contradicts the assumption that the strategy is optimal.

The proposition suggests that the optimality of the proposed strategy can be met by a proper choice of the frame length. That is, we can determine the frame length shorter than the average ON period, which is in practice a reasonable choice. On the other hand, a small frame size may result in poor utilization due to the frequent dedicated sensing. We numerically evaluate the impact of the frame length in Section IV. 


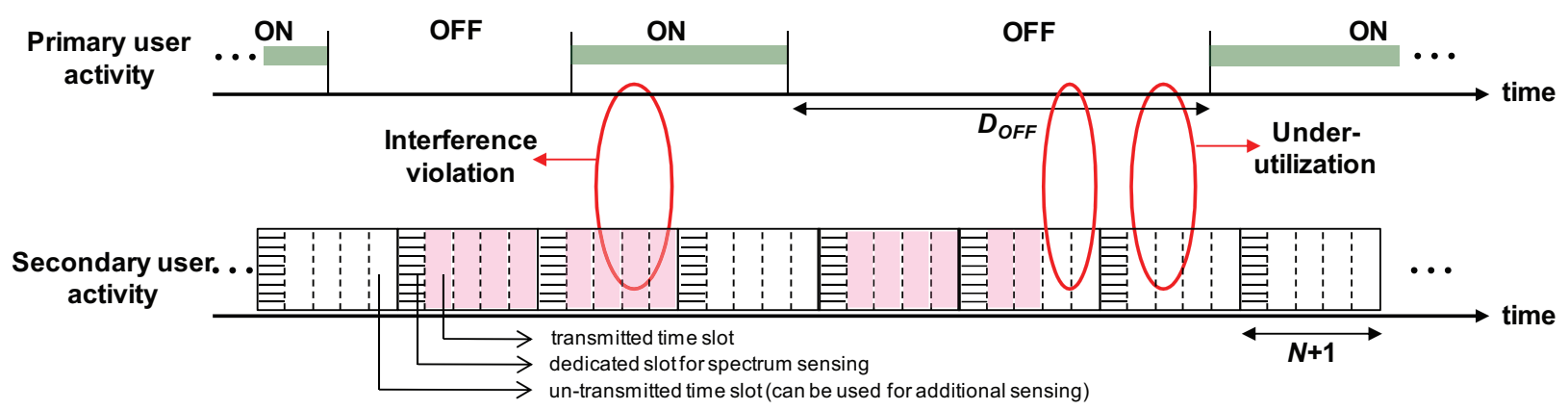

Fig. 1: The frame structure of the secondary user; interference violation and under-utilization according to the transmission strategy

From proposition 1, the optimization problem of (1)-(2) reduces to obtaining the optimal number of $n_{\tau}$. Let $\Psi_{i v}\left(n_{\tau}\right)$ be the expected number of interference violations during the frame given $n_{\tau}$. In order to obtain $\Psi_{i v}\left(n_{\tau}\right)$, we define an event $E_{\text {on }}(\tau+x)$ as follows:

- $E_{\text {on }}(\tau+x)$ is the event that the primary user begins the ON period at time slot $\tau+x$ given that it was idle at time slot $\tau$, i.e. $\sum_{k=1}^{x} I_{p u}(\tau+k)=1$ given that $I_{p u}(\tau)=0$. Then, $\Psi_{i v}\left(n_{\tau}\right)$ becomes $n_{\tau}$ if the event $E_{\text {on }}(\tau+1)$ occurs. On the other hand, no interference violation is expected during the $N$ time slots if $E_{o n}\left(\tau+n_{\tau}\right)$ does not occur. Thus, $\Psi_{i v}\left(n_{\tau}\right)$ is given by

$$
\Psi_{i v}\left(n_{\tau}\right)=\sum_{x=1}^{n_{\tau}} \operatorname{Pr}\left[E_{o n}(\tau+x)\right]\left(n_{\tau}+1-x\right) .
$$

At the decision moment $\tau, \operatorname{Pr}\left[E_{o n}(\tau+x)\right]$ in (4) can be obtained as follows:

$$
\begin{aligned}
\operatorname{Pr}\left[E_{\text {on }}(\tau+x)\right] & =\operatorname{Pr}\left(\tau+x-1 \leq D_{\text {off }}<\tau+x \mid D_{o f f}>\tau\right) \\
& =\frac{F_{o f f}(\tau+x)-F_{o f f}(\tau+x-1)}{1-F_{o f f}(\tau)}
\end{aligned}
$$

From (4) and (5),

$$
\begin{aligned}
\Psi_{i v}\left(n_{\tau}\right) & =\sum_{x=1}^{n_{\tau}} \frac{F_{o f f}(\tau+x)-F_{o f f}(\tau+x-1)}{1-F_{o f f}(\tau)}\left(n_{\tau}+1-x\right) \\
& =\frac{\sum_{x=1}^{n_{\tau}} F_{o f f}(\tau+x)-n_{\tau} F_{o f f}(\tau)}{1-F_{o f f}(\tau)}
\end{aligned}
$$

Now we consider a more relaxed model that the frame is not time-slotted such that the transmission time of the secondary user can be any real value within the frame. Let us denote the frame length by $U+T$ where $U$ is time required for the dedicated sensing and $T$ is the rest of the frame. At the moment $\tau$, the secondary user determines the duration $s_{\tau}$, during which it transmits $\left(0 \leq s_{\tau} \leq T\right)$. Similar to the slotted case, the secondary user transmits for $s_{\tau}$ seconds followed by $T-s_{\tau}$ seconds of no transmission. An extension of (6) is considered as a first step of obtaining the optimal $s_{\tau}$. Let us assume that a time slot is divided into logical sub-slots.
The duration of the sub-slot is denoted by $\Delta_{s}$. From the same reasoning to (4)-(6), we get

$$
\Psi_{i v}\left(s_{\tau}\right)=\frac{\sum_{x=1}^{s_{\tau} / \Delta_{s}} \Delta_{s} F_{o f f}\left(\tau+\Delta_{s} x\right)-s_{\tau} F_{o f f}(\tau)}{1-F_{o f f}(\tau)} .
$$

The summation in (7) can be converted to a definite integral as $\Delta_{s}$ approaches zero. From (2) and (7), the following constraint is obtained.

$$
\frac{\int_{0}^{s_{\tau}} F_{o f f}(\tau+x) d x-s_{\tau} F_{o f f}(\tau)}{1-F_{o f f}(\tau)} \leq T p_{t h r} .
$$

Then, the optimal $s_{\tau}$, say $s_{\tau}^{*}$, is obtained by finding the maximum $s_{\tau}$ which satisfies (8). Since the left hand side of (8) is a monotonically increasing function of the transmission time $s_{\tau}$, it can be readily solved by simple numerical methods such as bisection method in the range $[0, T]^{1}$.

It should be emphasized from (8) that $s_{\tau}^{*}$ depends on $\tau$, the elapsed time of the OFF period as well as $F_{\text {off }}(\cdot)$ which is coupled with the specific application. When the OFF duration $D_{o f f}$ follows an exponential distribution with a parameter $\lambda$, (8) reduces to

$$
\exp \left(-\lambda s_{\tau}\right)+\lambda s_{\tau} \leq \lambda T p_{t h r}+1 .
$$

Thus, $s_{\tau}^{*}$ is determined regardless of $\tau$ in case of the exponentially distributed OFF period due to the memoryless property of the exponential distribution.

\section{B. Impact of Sensing Error}

In practical environments, the detection of the primary activity may not be accurate. We consider two types of errors. First, the secondary user may not be aware of the accurate moment that the primary user begins the OFF period. This occurs when the secondary user relies only on the dedicated periodic sensing, and leads to the error of the maximum $T$ in the estimation of elapsed OFF duration. Let $\epsilon$ denote the difference between the perceived elapsed time $\tau$ and the actual one $\tau_{a}\left(\epsilon=\tau_{a}-\tau\right)$. Then, the expected interference violation

\footnotetext{
${ }^{1}$ For practical usage, a pre-calculated look-up table can be used so that the secondary user can refer it at the decision moments.
} 
during $T$ is denoted by $\Psi_{i v}\left(s_{\tau+\epsilon}\right)$. By letting $f_{\epsilon}(z)$ be the probability density function (PDF) of $\epsilon$, we get

$$
\Psi_{i v}\left(s_{\tau+\epsilon}\right)=\int_{0}^{T} \Psi_{i v}\left(s_{\tau+z} \mid \epsilon=z\right) f_{\epsilon}(z) d z .
$$

We assume that $\epsilon$ follows a uniform distribution in the range $[0, T]^{2}$. From (8) and (10), $s_{\tau}^{*}$ can be obtained by maximizing $s_{\tau}$ in the following formula:

$\int_{0}^{T}\left(\frac{\int_{0}^{s_{\tau}} F_{o f f}(\tau+z+x) d x-s_{\tau} F_{o f f}(\tau+z)}{1-F_{o f f}(\tau+z)}\right) \frac{1}{T} d z \leq T p_{t h r}$

The second type of error is false alarm and missed detection due to the unreliable spectrum sensing. We consider the missed detection in this study because it leads to much adverse impact. Let $p_{m}$ be the probability that the secondary user detects the primary user as idle while it is actually busy. The parameter $p_{m}$ is specified by the receiver operating characteristic (ROC) curve of a sensing technique [10]. Since $s_{\tau}$ seconds of the interference violation occurs in case of the missed detection, the optimal $s_{\tau}$ in the presence of the missed detection, $s_{\tau, m}^{*}$, is given by

$$
s_{\tau, m}^{*}=s_{\tau}^{*}\left(1-p_{m}\right)+0 p_{m}=s_{\tau}^{*}\left(1-p_{m}\right) .
$$

This means that the presence of the missed detection results in a conservative decision, i.e. a reduced transmission time.

\section{NumericAl RESUlts}

\section{A. Source Traffic Model for the Primary User}

The duration of the OFF period $D_{\text {off }}$ can be modeled by a probability distribution depending on the application of the primary user. We consider two source traffic models for the primary user: $\mathrm{P} 2 \mathrm{P}$ and interactive gaming. For the $\mathrm{P} 2 \mathrm{P}$ service, $D_{o f f}$ is described by a log-normal distribution [20]. The PDF of $D_{o f f}$ is given by

$$
f_{o f f}(t ; \mu, \sigma)=\frac{1}{t \sigma \sqrt{2 \pi}} e^{\frac{-(\ln t-\mu)^{2}}{2 \sigma^{2}}} .
$$

The OFF period of the interactive gaming is modeled by an extreme value distribution with the following PDF [21], [22].

$$
f_{o f f}(t ; \mu, \sigma)=\frac{1}{\sigma} e^{-\frac{t-\mu}{\sigma}} e^{e^{-\frac{t-\mu}{\sigma}}} .
$$

The parameters $(\mu, \sigma)$ are in milliseconds (msec) and the values used in this paper are $(2.47,1.88)$ and $(55.00,6.00)$ for the P2P and the interactive gaming, respectively [20], [22]. Note that the parameters give the mean OFF duration of 69.10 msec and standard deviation of $396.18 \mathrm{msec}$ for the P2P, and $51.50 \mathrm{msec}$ and $7.70 \mathrm{msec}$ respectively for the interactive gaming.

The PDFs of these traffic models represent different characteristics as shown in Fig. 2. The P2P is well fitted by heavy tail

\footnotetext{
${ }^{2}$ We verified the assumption through Monte Carlo simulation, though the result does not appear in the paper.
}

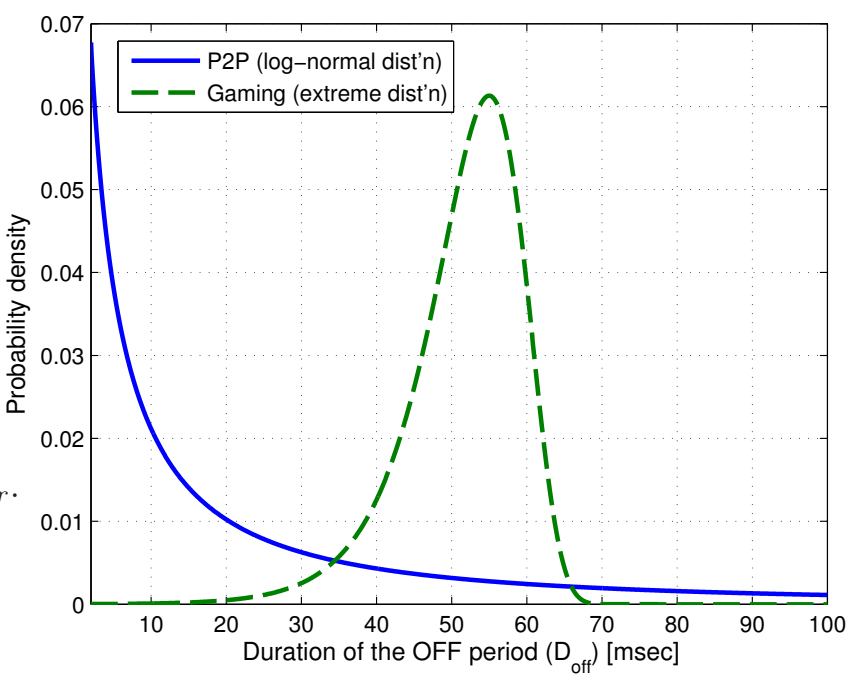

Fig. 2: PDFs of $D_{\text {off }}$ for the P2P and the interactive gaming

distributions. The OFF durations for various Internet services can be described by the log-normal distribution [20]. On the other hand, the interactive gaming shows a spiky shape with a small standard deviation compared to the P2P.

The relationship between the elapsed time of the OFF period and the remaining OFF duration is characterized as shown in Fig. 3. This figure depicts $\operatorname{Pr}\left[E_{\text {on }}(\tau+1)\right]$ for each traffic model. Recall that $\operatorname{Pr}\left[E_{\text {on }}(\tau+1)\right]$ denotes the probability that the primary user begins the busy period at $\tau+1$ given that it was idle at $\tau$. From $(5), \operatorname{Pr}\left[E_{\text {on }}(\tau+1)\right]$ at $\tau$ is given by

$$
\operatorname{Pr}\left[E_{\text {on }}(\tau+1)\right]=\frac{F_{\text {off }}(\tau+1)-F_{\text {off }}(\tau)}{1-F_{\text {off }}(\tau)} .
$$

The exponential distribution which has the same mean OFF duration is also shown for comparison. It is interesting to observe opposite characteristics of the $\mathrm{P} 2 \mathrm{P}$ and the interactive gaming in terms of the remaining OFF period. Fig. 3 indicates that the OFF period tends to continue as $\tau$ increases in the $\mathrm{P} 2 \mathrm{P}$ service, whereas longer elapsed time results in higher probability of ending the idle period in the interactive gaming. For the case of the exponential distribution, $\operatorname{Pr}\left[E_{\text {on }}(\tau+1)\right]$ has the same value regardless of $\tau$.

From (8) and (12), the optimal $s_{\tau}$ for each traffic model is obtained in Fig. 4. As expected from Fig. 3, the P2P and the interactive gaming show opposite trends in the relationship between the elapsed OFF period and $s_{\tau, m}^{*}$. It is observed that $s_{\tau, m}^{*}$ for the P2P increases as the elapsed time proceeds, while $s_{\tau, m}^{*}$ decreases according to the increase in $\tau$ for the interactive gaming. This figure suggests that the transmission strategy of the secondary user should be adapted to not only the elapsed idle period but also the characteristics of the primary user traffic.

\section{B. Performance Measures}

We define the interference violation probability $p_{i v}$ and the spectrum utilization $\Phi$ as performance metric. The number of dedicated spectrum sensings in an OFF period is denoted 


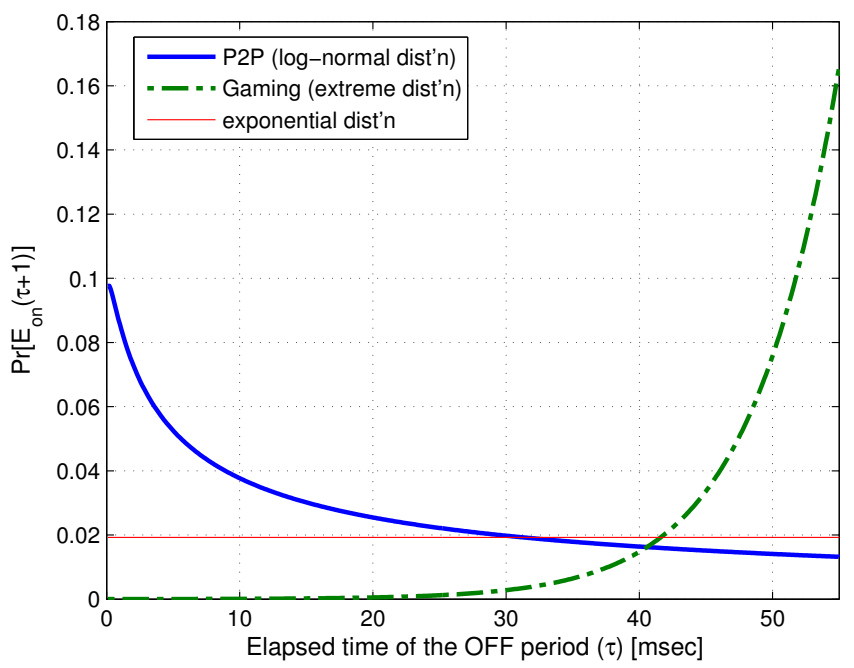

Fig. 3: $\operatorname{Pr}\left[E_{\text {on }}(\tau+1)\right]$ for the P2P, interactive gaming, and exponential distribution

by $\eta_{d s}$. Let $V_{i v}$ be the time of interference violation during the OFF period, and $V_{s u c}$ be the time that the secondary user transmits without interference violation. From above notations, $p_{i v}$ and $\Phi$ are given by

$$
\begin{gathered}
p_{i v}=\frac{\mathbf{E}\left[V_{i v}\right]}{\mathbf{E}\left[D_{o f f}\right]-U \mathbf{E}\left[\eta_{d s}\right]}, \\
\Phi=\frac{\mathbf{E}\left[V_{s u c}\right]}{\mathbf{E}\left[D_{o f f}\right]},
\end{gathered}
$$

where $\mathbf{E}[\cdot]$ denotes the expected value. We also define $\Phi_{\max }$ to be the hypothetically maximum achievable utilization.

$$
\Phi_{\max }=\frac{\mathbf{E}\left[D_{o f f}\right]-U \mathbf{E}\left[\eta_{d s}\right]}{\mathbf{E}\left[D_{o f f}\right]} .
$$

For the comparison purpose, a simple transmission strategy, namely exponential approximation is considered where $D_{o f f}$ is assumed to follow the exponential distribution $(\lambda=$ $\left.1 / \mathbf{E}\left[D_{o f f}\right]\right)$.

\section{Simulation Results}

Monte Carlo simulations are performed to examine the performance measures defined in Section IV-B. Throughout the experiments, the dedicated sensing duration $U$ is assumed to be $1.0 \mathrm{msec}$. Fig. 5 shows $p_{i v}$ as a function of $T$, the maximum continuous transmission time for the secondary user. The exponential approximation does not satisfy the $p_{t h r}$ requirement for both $\mathrm{P} 2 \mathrm{P}$ and interactive gaming. This indicates that the use of a Markovian assumption generates excess interference to the primary user in practical environments. The increase in $p_{i v}$ is observed in the $\mathrm{P} 2 \mathrm{P}$ case as $T$ increases. The prediction of the primary user activity becomes inaccurate with the increasing frame length. The impact of $\epsilon$, the error in the estimation of the elapsed OFF duration, also increases with longer $T$. As a result, $p_{i v}$ exceeds $p_{t h r}$ as $T$ becomes longer than $8 \mathrm{msec}$ in the presence of uniformly distributed $\epsilon$, and 12 msec without $\epsilon$. On the other hand, the effect of $T$ on $p_{i v}$ is

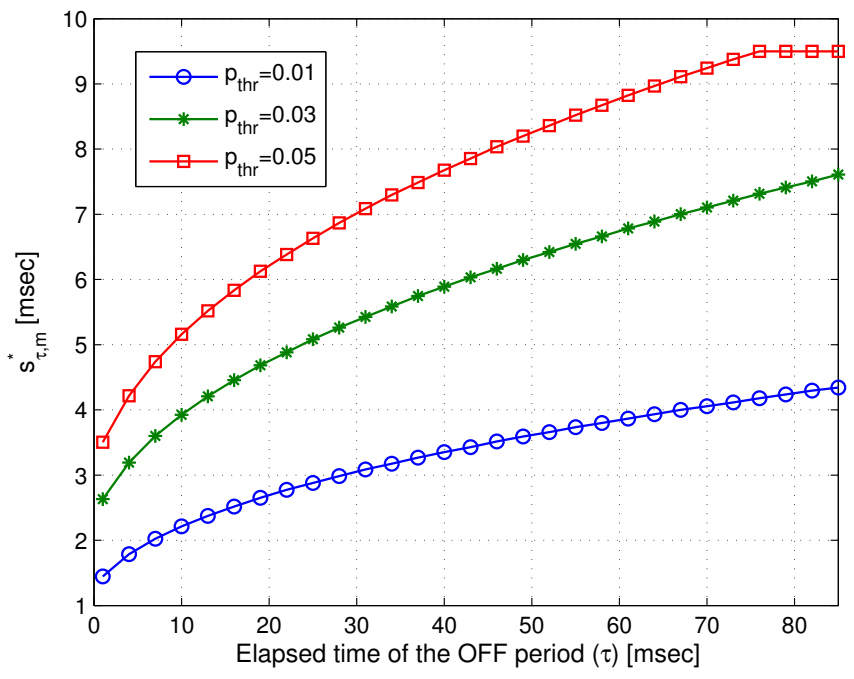

(a) $\mathrm{P} 2 \mathrm{P}$

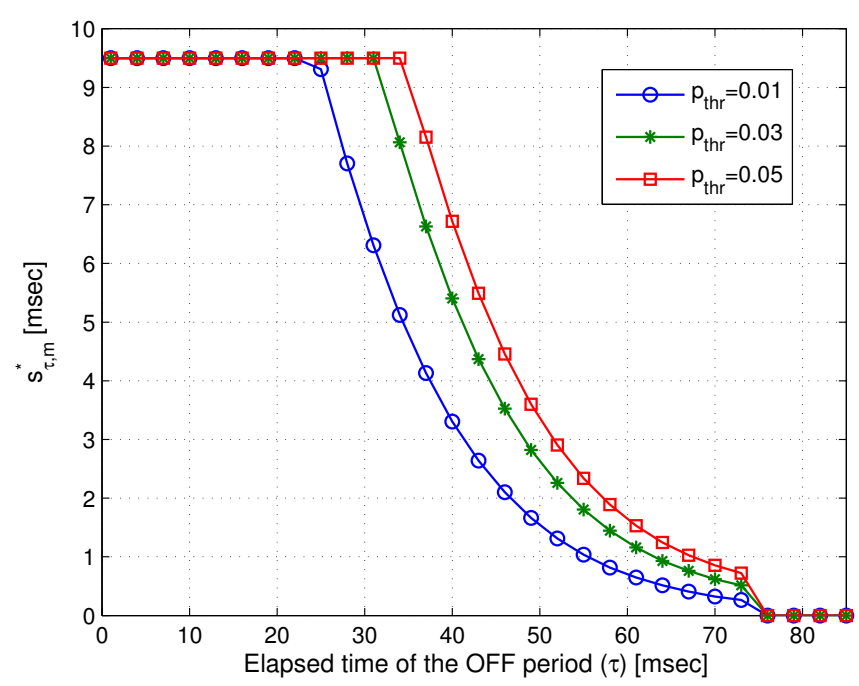

(b) Interactive gaming

Fig. 4: $s_{\tau, m}^{*}$ as a function of $\tau\left(T=10 \mathrm{msec}, p_{m}=0.05\right.$, and $\epsilon=0)$

small for the case of the interactive gaming, which shows the gaming is not sensitive to the frame length. It is because the extreme value distribution describing the interactive gaming has a small standard deviation.

The utilization $\Phi$ according to $T$ is presented in Fig. 6 . The result of the exponential approximation is not shown in the figure because the approximation does not satisfy $p_{t h r}$ requirement. The gap between $\Phi$ and $\Phi_{\max }$ increases as $T$ becomes larger due to the increasing inaccuracy in predicting the remaining OFF duration of the primary user. For the case of P2P, the longer frame even decreases the utilization. Overall, the interactive gaming gives better $\Phi$ than the P2P. It can be explained by the fact that the interactive gaming has a smaller standard deviation, which makes accurate prediction of the primary user activity available.

The relationship between $p_{t h r}$ and $\Phi$ is illustrated in Fig. 7. The $p_{t h r}$ constraint can be regarded as the tolerance of the per- 


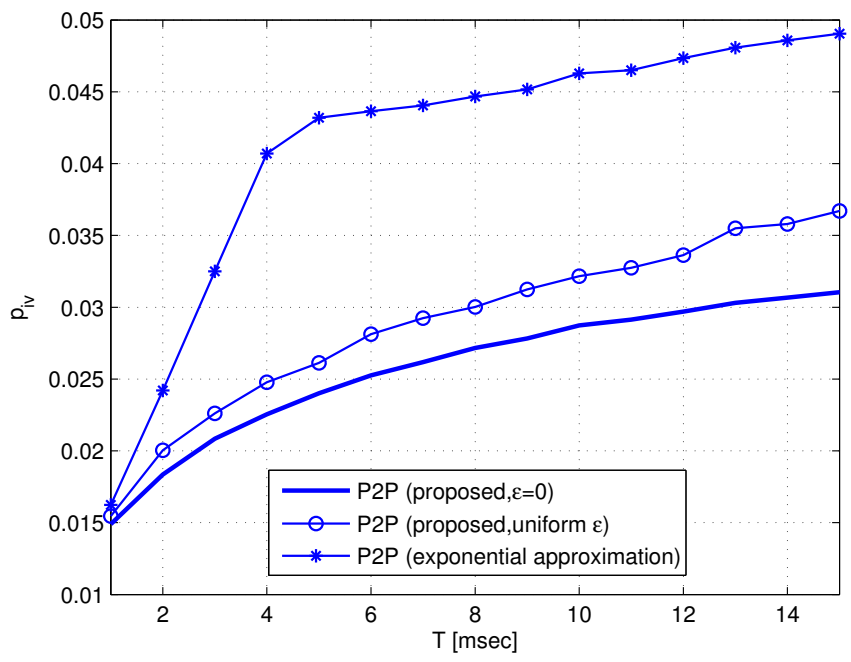

(a) $\mathrm{P} 2 \mathrm{P}$

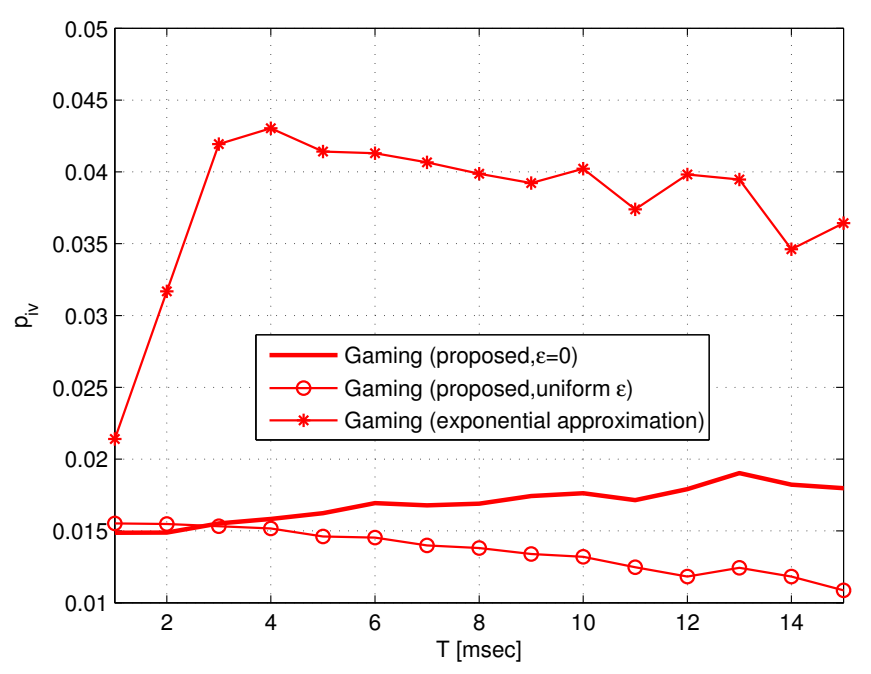

(b) Interactive gaming

Fig. 5: $p_{i v}$ as a function of $T\left(p_{t h r}=0.03\right.$ and $\left.p_{m}=0.05\right)$

formance impairment that the primary user experiences. Thus, Fig. 7 shows an important tradeoff between the performance degradation of the primary user and the utilization of the secondary user. The increase in $p_{t h r}$ improves $\Phi$ significantly when $p_{t h r}$ is relatively small, i.e. less than 0.03 for the P2P and 0.01 for the interactive gaming. However, the rate of the performance improvement is reduced as $p_{t h r}$ increases. This implies that the proper choice of $p_{t h r}$ will be beneficial to both the primary and the secondary users. Moreover, the choice of $p_{t h r}$ should depend on the application that the primary user employs. For the case of the P2P, a shorter $T$ gives better $\Phi$ when $p_{t h r}$ is lower than 0.07 . Thus, smaller frame size is preferred if a tight requirement for the interference violation is applied.

Finally, Fig. 8 shows the change in $p_{i v}$ when there occurs an error in the parameter estimation of the probability distribution for the primary source traffic. We assume that $\mu$ and $\sigma$ in (13) and (14) have the same relative error. The over (under)estimation of the parameters means the secondary user estimates $\mathbf{E}\left[D_{o f f}\right]$ longer (shorter) than actual OFF duration. For

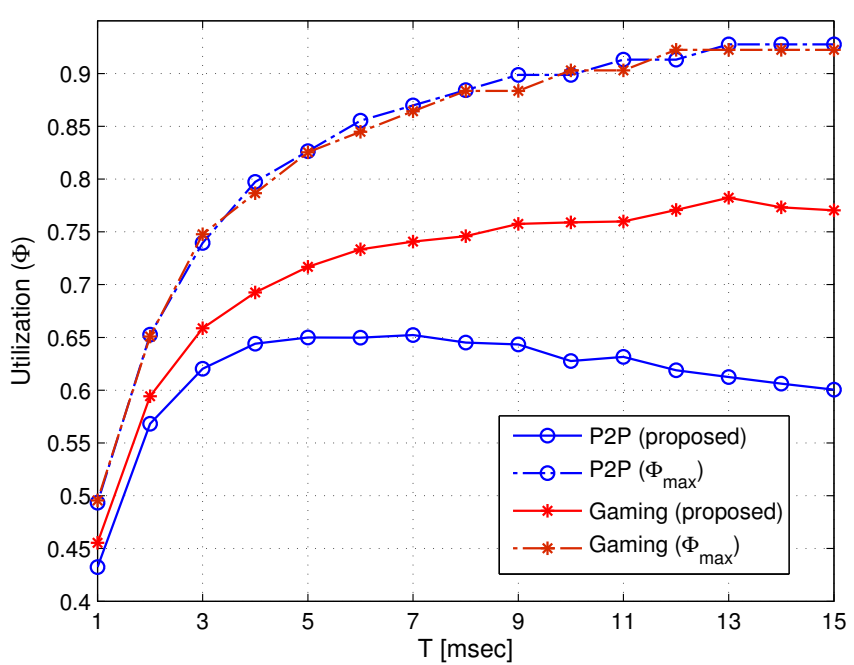

Fig. 6: $\Phi$ as a function of $T\left(p_{t h r}=0.03, p_{m}=0.05\right.$, and $\epsilon=0$ )

both of the $\mathrm{P} 2 \mathrm{P}$ and the interactive gaming, under-estimation of the traffic parameter results in lower $p_{i v}$ while over-estimated parameters cause excess interference violations. In practice, there is an uncertainly in the parameter estimation when the primary user begins a new application. The secondary user can estimate the parameters more precisely as the application continues. Therefore, it is recommended that the secondary user make a conservative decision on the traffic parameters when a change in the traffic distribution is detected.

\section{CONCLUSION}

We investigated the temporal aspect of OSA. Instead of the traditional Markovian models, realistic ON/OFF traffic models, better adapted to the bursty nature of the traffic, were considered. Knowledge about the elapsed OFF period of the primary user is exploited to obtain a prediction of the remaining OFF duration that controls the secondary user behavior. We considered a frame structure where the secondary user determines its transmission power at the beginning of each frame based on the prediction of the primary user activity. An optimal strategy of the secondary user was proposed, which can be readily adapted to any primary traffic model.

Our findings on interactive gaming and P2P primary traffic are as follows: the OFF duration of the gaming has a spikyshaped PDF while that of the P2P follows a heavy-tailed distribution. As for the gaming, the secondary user has to reduce the transmission power as the OFF period of the primary user elapses. This means, as the duration of the OFF period is centered to a certain value with high probability, the secondary user should turn off its power immediately after a given time. The opposite applies to the P2P. The simulation results show that the interference violation is well bounded by the proposed scheme for both traffic models.

The results also provide insights into the research issues in the temporal OSA. First, the performance can further improve by a proper choice of the frame length. The gaming is not sensitive to the frame size of the secondary user. On the other 


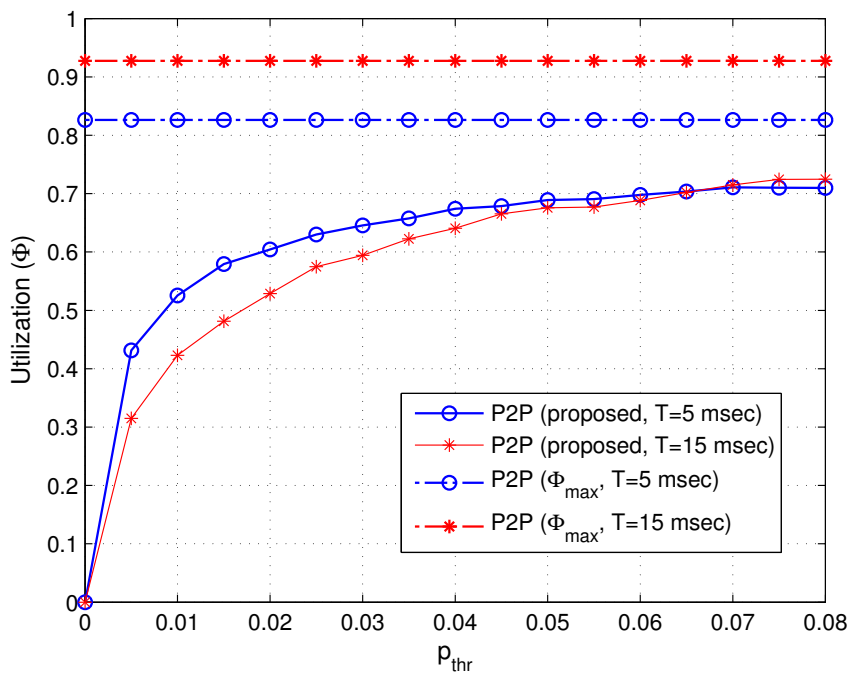

(a) $\mathrm{P} 2 \mathrm{P}$

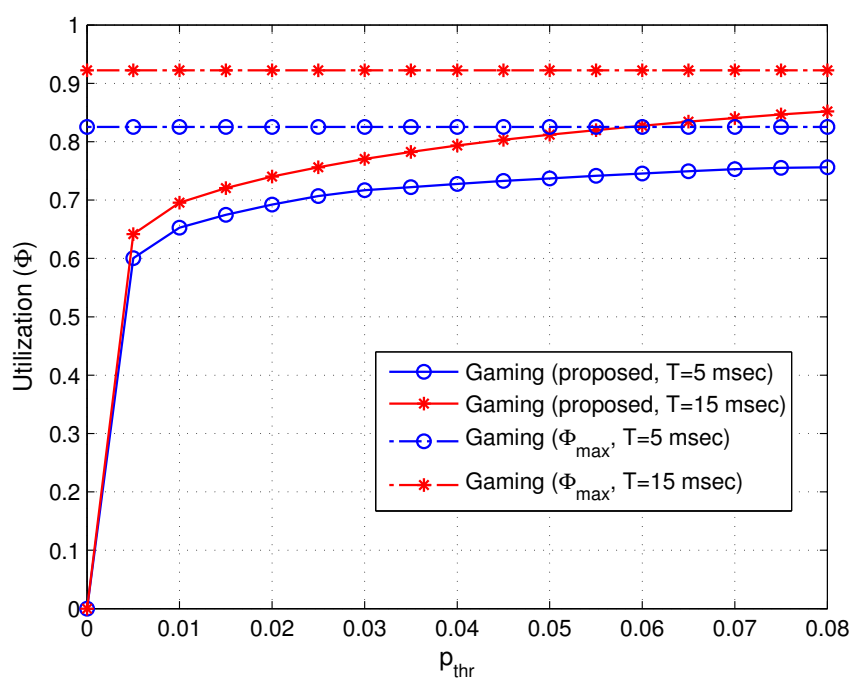

(b) Interactive gaming

Fig. 7: $\Phi$ as a function of $p_{t h r}\left(p_{m}=0.05\right.$ and $\left.\epsilon=0\right)$

hand, the heavy-tailed services like P2P needs an accurate estimation of the remaining OFF duration, requiring smaller frame length. Second, the constraint on the interference violation plays an important role in optimizing both primary and secondary performances. Third, an over-estimation of the traffic parameters results in high probability of the interference violation. Therefore, the following topics remain as attractive areas of further studies: the determination of frame length, the performance balancing between the primary and the secondary users, and the estimation of the traffic distribution and parameters.

\section{REFERENCES}

[1] Federal Communications Commission, Spectrum Policy Task Force, "Report of the Spectrum Efficiency Working Group," Nov. 2002, [Online]. Available: http://www.fcc.gov/sptf/reports.html.

[2] M. A. McHenry, P. A. Tenhula, D. McCloskey, D. A. Roberson, and C. S. Hood, "Chicago Spectrum Occupancy Measurements \& Analysis and a Long-term Studies Proposal," in Proc. 1st International Workshop on Technology and Policy for Accessing Spectrum (TAPAS '06), 2006.

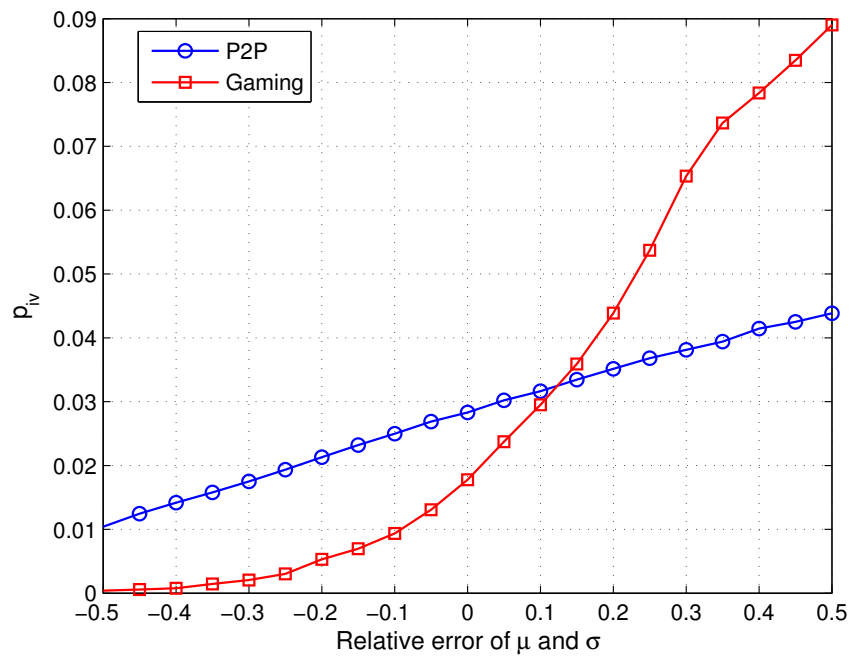

Fig. 8: $p_{i v}$ as a function of the relative error in $\mu$ and $\sigma(T=$ $10 \mathrm{msec}, p_{t h r}=0.03, p_{m}=0.05$, and $\epsilon=0$ )

[3] J. Mitola III and G. Q. Maguire Jr, "Cognitive Radio: Making Software Radios More Personal," IEEE Personal Commun. Mag., vol. 6, no. 4, pp. 13-18, Aug. 1999.

[4] J. Mitola III, "Cognitive Radio: An Integrated Agent Architecture for Software Defined Radio," Ph.D. dissertation, Royal Institute of Technology (KTH), Stockholm, Sweden, May 2000.

[5] A. Ghasemi and E. S. Sousa, "Fundamental Limits of Spectrum-Sharing in Fading Environments," IEEE Trans. Wireless Commun., vol. 6, no. 2, pp. 649-658, Feb. 2007.

[6] S. Srinivasa and S. A. Jafar, "How Much Spectrum Sharing is Optimal in Cognitive Radio Networks?" IEEE Trans. Wireless Commun., vol. 7 , no. 10 , pp. 4010-4018, Oct. 2008.

[7] R. Menon, R. M. Buehrer, and J. H. Reed, "On the Impact of Dynamic Spectrum Sharing Techniques on Legacy Radio Systems," IEEE Trans. Wireless Commun., vol. 7, no. 11, pp. 4198-4207, Nov. 2008.

[8] E. G. Larsson and M. Skoglund, "Cognitive Radio in a FrequencyPlanned Environment: Some Basic Limits," IEEE Trans. Wireless Commun., vol. 7, no. 12, pp. 4800-4806, Dec. 2008.

[9] J. Zander, "Can We Find (and Use) "Spectrum Holes"? Spectrum Sensing and Spatial Reuse Opportunities in "Cognitive" Radio Systems," in Proc. 69th IEEE Vehicular Technology Conference (VTC), Barcelona, Apr. 26-29 2009.

[10] Q. Zhao and B. M. Sadler, "A Survey of Dynamic Spectrum Access: Signal Processing, Networking, and Regulatory Policy," IEEE Signal Process. Mag., vol. 24, no. 3, pp. 79-89, May 2007.

[11] Q. Zhao, L. Tong, A. Swami, and Y. Chen, "Decentralized Cognitive MAC for Opportunistic Spectrum Access in Ad Hoc Networks: A POMDP Framework," IEEE J. Sel. Areas Commun., vol. 25, no. 3, pp. 589-600, Apr. 2007.

[12] Q. Zhao, S. Geirhofer, L. Tong, and B. M. Sadler, "Opportunistic Spectrum Access via Periodic Channel Sensing," IEEE Trans. Signal Process., vol. 56, no. 2, pp. 785-796, Feb. 2008.

[13] L. Yang, L. Cao, and H. Zheng, "Proactive Channel Access in Dynamic Spectrum Networks," Physical Communication, vol. 1, no. 2, pp. 103$111,2008$.

[14] M. G. Khoshkholgh, K. Navaie, and H. Yanikomeroglu, "On the Impact of the Primary Network Activity on the Achievable Capacity of Spectrum Sharing over Fading Channels," IEEE Trans. Wireless Commun., vol. 8, no. 4, pp. 2100-2111, Apr. 2009.

[15] W.-Y. Lee and I. F. Akyildiz, "Optimal Spectrum Sensing Framework for Cognitive Radio Networks," IEEE Trans. Wireless Commun., vol. 7, no. 10 , pp. $3845-3857$, Oct. 2008.

[16] H. Kim and K. Shin, "Efficient Discovery of Spectrum Opportunities with MAC-Layer Sensing in Cognitive Radio Networks," IEEE Trans. Mobile Comput., vol. 7, no. 5, pp. 533-545, May 2008.

[17] X. Zhou, J. Ma, G. Y. Li, Y. H. Kwon, and A. C. K. Soong, "ProbabilityBased Optimization of Inter-Sensing Duration and Power Control in Cognitive Radio," IEEE Trans. Wireless Commun., vol. 8, no. 10, pp. 4922-4927, Oct. 2009. 
[18] P. Tran-Gia, D. Staehle, and K. Leibnitz, "Source Traffic Modeling of Wireless Applications," AEU - International Journal of Electronics and Communications, vol. 55, no. 1, pp. 27-36, 2001.

[19] S. Geirhofer, L. Tong, and B. Sadler, "Dynamic Spectrum Access in the Time Domain: Modeling and Exploiting White Space," IEEE Commun. Mag., vol. 45, no. 5, pp. 66-72, May 2007.

[20] A. Klemm, C. Lindemann, and M. Lohmann, "Traffic Modeling and Characterization for UMTS Networks," in Proc. IEEE Global Telecommunications Conference (GLOBECOM), vol. 3, Nov. 25-29 2001, pp. 1741-1746.

[21] M. S. Borella, "Source Models of Network Game Traffic," Computer Communications, vol. 23, no. 4, pp. 403-410, 2000.

[22] J. Färber, "Network Game Traffic Modelling," in Proc. 1st ACM Workshop on Network and System Support for Games (NetGames), Apr. 16-17 2002, pp. 53-57. 